\title{
The mechanism of non-numerical anchoring heuristic based on magnitude priming: is it just the basic anchoring effect in disguise?
}

\begin{abstract}
The anchoring heuristic refers to phenomena when an arbitrary number affects subsequent numerical estimations. Oppenheimer, LeBoeuf and Brewer (2008) showed that it is not necessary for the anchor to be a numerical value (i.e., the act of drawing lines of different length effectively shifts numerical estimations), yet current models describing the anchoring heuristic do not fully account for the mechanism of non-numerical anchoring. However, this effect shows similarity to the basic anchoring effect-obtained without the comparative question and based on the availability of the given number in working memory. In this study, we attempt to verify whether those two effect share the same psychological mechanism. In Experiment 1, we show that non-numerical anchoring based on magnitude priming cannot be obtained when the lines are just observed. The examined mechanism proves to be dependent on the act of drawing, displaying limitations similar to the basic anchoring effect, previously pointed out by Brewer and Chapman (2002). By using the same numerical anchors in different size formats, in Experiment 2 we showed that anchoring based on magnitude priming occurs even when the numerical values do not affect the estimations. The results are discussed in the light of a possible mechanism that underlies the investigated effect.
\end{abstract}

Key words: anchoring effect, magnitude priming, non-numerical anchoring, estimations

\section{Introduction}

When making numerical judgments in everyday life we try to produce the best possible answer based on accessible information. However, precise and objective numerical information is often unavailable. Under such conditions, individuals are likely to seek other numerical clues to aid judgments and decision making. To give an example, imagine walking into a grocery store. There is a stand with fruit: apples, pears, plums and many more. Each kind of fruit has its own price, yet the price for the apples is missing. How much do the apples cost? Is the price just the same as for the other kinds of fruit, or maybe their price should be higher or lower? Numerous research (e.g. Tversky \& Kahneman, 1974; Gilovich, Griffin, \& Kahneman, 2002; for review see Furnham \& Boo, 2011) has repeatedly documented that if we are not certain about the correct answer and the necessary information is not given, we are prone to base our judgment on irrelevant cues. The anchoring heuristic describes how numerical estimations may be shifted as a result of presenting an arbitrary number. However, recent research has shown that not only numbers affect our numerical estimations - the cross-modal anchoring (Oppenheimer, LeBoeuf, \& Brewer, 2008) refers to phenomena in which the estimations are biased because of drawing lines of different length. It is difficult to account for this effect by using the theoretical framework explaining the numerical anchoring, however, this mechanism shows similarities to a mechanism called the basic anchoring. The main goal of this study is to verify whether it is the same psychological mechanism that underlies those two effects.

\footnotetext{
" SWPS University of Social Sciences and Humanities, Wroclaw Faculty of Psychology, Ostrowskiego 30b, 53-238 Wroclaw, Poland

Corresponding author: Pawel Tomczak, e-mail: ptomczak1@swps.edu.pl
}

Authors of this manuscript report no conflicts of interest in relation to the submitted manuscript. 
In the next sections we will describe the anchoring heuristic and present the mechanisms responsible for this effect. Then, we will proceed to the different methods of anchoring: the basic anchoring and non-numerical anchoring. Finally, we summarize the current state of knowledge about the anchoring heuristic and present our research questions.

\section{Anchoring heuristic}

In their seminal study, Tversky and Kahneman (1974) asked people about the percentage of African countries belonging to the United Nations. Just before the main question, participants had to answer whether the target number is higher or lower than a randomly generated number ranging from 1 to 100 . Even though the generated numbers were completely irrelevant, participants who received relatively larger numbers gave higher estimates than participants who received relatively smaller numbers. In the case of percentages, numbers ranging from 1 to 100 seem like plausible answers. This could, in fact, explain why the estimations were biased. Yet, extreme values have also been proven to anchor the estimations effectively (Strack \& Mussweiler, 1999). Even when using anchor values that are not a reasonable answer to the initial question (i.e. numbers 1952 and -300 when asking about the year of birth of Leonardo da Vinci) the anchoring effect still occurs.

Interestingly, even professional expertise is not enough to avoid the effect of the anchoring heuristic. For example, Northcraft and Neal (1987) asked real estate agents to appraise the value of a house. They allowed them to examine the place and its surroundings. Despite the fact that all of the real estate agents had to appraise the same property with the same facilities, their estimations varied significantly depending on the listing price provided. If the listing price was high, the house was appraised as more valuable than the same house but with a lower listing price. This effect can be also observed in areas such as clinical judgments (Friedlander \& Stockman, 1983), negotiations (Kristensen \& Garling, 1997) or expectancy of return in the stock market (Kaustia, Alho, \& Puttonen, 2008). What is then, the psychological mechanism that underlies judgment based on the anchoring heuristic?

\section{Mechanisms of anchoring}

Research provides several possible explanations of the anchoring heuristic. Tversky and Kahneman (1974) claim that the number serving as the anchor is considered as a starting point for estimating the target value. After the comparative question it is necessary to adjust from the provided anchor in search for a possible answer. Using a high anchor results in an adjustment towards lower values, while low anchor entails to focus on higher values. The adjustment process is considered to continue until the range of plausible values is reached (Quattrone, Lawrence, Finkel, \& Andrus, 1984). As a result of insufficient adjustment, the process stops within the upper or lower limit of the range. In effect, the subsequently generated value is biased towards the anchor (Tversky \& Kahneman, 1974; Epley \& Gilovich, 2001).
An alternative explanation is the selective accessibility model (Strack \& Mussweiler, 1997). Here, the anchoring mechanism is considered to be a consequence of processes based on positive-hypothesis testing (Klayman \& Ha, 1987). As a result of the comparative question, the first considered hypothesis focuses on the possibility that the anchor value may be in fact a correct answer. The information supporting this hypothesis is selectively chosen and taken into account. In effect, provided that this knowledge is perceived as applicable and relevant, it becomes more accessible and it is used in the decision process (Higgins, Rholes, \& Jones, 1977; Higgins \& Brendl, 1995). Given that the evidence supporting the hypothesis seems to be substantial, the final estimations are biased towards the anchor value. According to Mussweiler and Strack (1999) it is crucial that the anchor and the estimation target relate to the same dimension. For example, considering whether the Brandenburg Gate is higher or lower than 150 meters anchors subsequent estimations of height, but does not affect the estimations regarding width.

\section{Basic anchoring}

Interestingly, explanations of the anchoring effect mostly refer to the mechanism based on asking a comparative question before giving the absolute answer. Kahneman and Knetsch (1993) proposed that the comparative question is not necessary. Storing a number in short-term memory is sufficient to observe the anchoring effect. During the judgment the number in short-term memory is brought to mind automatically and considered as a possible answer despite its source. Wilson, Houston, Brekke and Etling (1996) showed that copying a list of numbers or performing computations shifted following estimations. Yet, the replication of this effect (Brewer \& Chapman, 2002) showed that basic anchoring is highly proceduredependent and not as robust as the anchoring based on the comparative question. Simple procedural changes such as changing the order of presentation of the anchors disrupted the expected effect. Moreover, copying a list of numbers was not enough to anchor the subsequent estimations. However, the anchoring effect was observed after five pages of computational tasks and when the anchors resembled dates (i.e. 1928) in comparison to other numbers (for example, resembling postal codes). As stated by Brewer and Chapman (2002), the generalizability of the basic anchoring is quite limited.

\section{Non-numerical anchoring}

On the contrary to previous findings, further research proves that the anchoring effect without a comparative question is quite possible and robust when one change is applied - using physical stimuli instead of numerical anchors. LeBoeuf and Shafir (2006) showed, that using physical stimuli effectively anchors estimations regarding physical attributes such as length, weight or loudness. For example, participants had to examine a line of a certain length. Then, they had to recreate it either by trimming a longer line or lengthening a shorter one. As a result of insufficient adjustment the final line was too long in 
the trimming condition or too short in the lengthening condition. It is important to mention, that in the study conducted by LeBoeuf and Shafir (2006) participants could inspect the target of the estimations (for example, when asked about weight they were allowed to pick up the actual object that was the target of the estimations). A recent study by Oppenheimer, LeBoeuf and Brewer (2008) reveals that physical anchors also affect the estimations regarding general knowledge: participants who drew long lines made higher estimations regarding the length of the Mississippi river than participants who drew short lines. In this particular case of anchoring, the estimations were presumably shifted due to the sense of "largeness" or "smallness" generated by drawing the lines (Oppenheimer et al., 2008). Interestingly, drawing lines anchored not only estimations regarding length but also temperature.

It is substantial to note that in the procedure used by LeBoeuf and Shafir (2006) the goal of the task was clear for the whole time, possibly affecting the cognitive processes engaged in processing the stimuli. Despite that Oppenheimer et al. (2008) did not mention the estimation target during the anchoring task, both procedures demanded focusing on similar spatial attributes such as length and shape. In experiment 1 we aimed to answer: will the anchoring effect be acquired when the lines are being observed without any cues?

Strack and Mussweiler (1997) claim that the anchor has to be compatible with the target of estimations, while results of Oppenheimer et al. (2008) show cross-modality of nonnumerical anchoring. In this regard non-numerical anchoring resembles the robustness of classical anchoring, where only the absolute values of the anchor are crucial in shifting the estimations (Wong \& Kwong, 2000). Yet, the cross-modal anchoring effect is acquired without asking the comparative question, similar to the basic anchoring. How to explain the fact that unrelated physical stimuli affect numerical estimations? In Experiment 1 we attempt to establish what is the relation between physical stimuli, such as lines, and numerical estimations regarding general knowledge. Is it possible to predict the magnitude of the estimates based on the length of the drawn lines represented in millimeters?

\section{Present study}

The study conducted by Oppenheimer, LeBoeuf and Brewer (2008) shows that the anchoring effect does not have to rely on numbers serving as anchors. The mechanisms provided by current studies are not suitable for explaining this effect since there is no point of reference for adjustment (Tversky \& Kahneman, 1974; Epley \& Gilovich, 2001). Also, the cross-modal anchoring does not require compatibility between the anchor and the estimation task, postulated by Strack and Mussweiler (1997). Similar to basic anchoring, no comparative question is needed to anchor the estimations, although basic anchoring suggests considering the number stored in shortterm memory as a plausible answer. Lines are simply not numbers, hence this model cannot explain how the nonnumerical anchoring actually biases the estimations. In this paper we attempt to verify the limits of non-numerical anchoring. In Experiment 1, we investigate whether the act of drawing is crucial to generating a sense of "largeness" and "smallness" in order to shift the estimations regarding general knowledge. Basing our predictions in the results obtained by Brewer and Chapman (2002) we expect that observing the lines attentively would not lead to the same results as when the lines are drawn: observing the lines without any cues will not affect subsequent estimations. Additionally, we hypothesize that the actual length of the drawn lines represented in millimeters will have its reflection in the magnitude of the estimations.

\section{Experiment 1}

There are two crucial aspects of the cross-modal anchoring that we wanted to establish in this experiment. First, whether observing the lines without explicit instructions about the estimation task will shift the subsequent estimations regarding general knowledge. Second, what is the relation between the length of the drawn lines and the magnitude of estimations. In order to answer our empirical questions we conducted a study similar to Oppenheimer et al. (2008) using a computerbased procedure. Our experiment was a 2 (drawing or observing) x2 (short or long lines) design. Regarding the drawing condition, we expect to replicate the findings from previous studies. Therefore, we hypothesize that an average estimate in the long lines condition will be higher than in the short lines condition (H1).

In case of the observing condition, we expect that observing the lines should not suffice to shift the estimations effectively: there will be no difference between the average estimates (H2). We expect that without a clear purpose for observing the lines, such as copying them (Oppenheimer et al., 2008) or further reproduction (LeBoeuf \& Shafir, 2006), observing the lines will not affect the estimation process.

Also, we hypothesize that the estimations do not result directly from the presented lines but are determined by the length of the lines that are drawn. The length of drawn lines will mediate the relation between the lines condition and estimates $(\mathrm{H} 3)$.

\section{Method}

\section{Participants}

One hundred and forty-eight psychology students participated in the experiment in exchange for course credits. Participants provided informed consent before the experiment. The participation in this study was voluntary, anonymous, and in agreement with the guidelines of the Ethical Committee.

\section{Procedure, stimuli and design}

The experiment took place in a computer room where participants were assigned in turns to a drawing or an observing condition. Within the condition, participants were randomly assigned to a short or long lines condition. We used two sets of three lines, previously used by Oppenheimer et al. (2008) (Long lines: 21 centimeters; short lines: 6 centimeters; Fig. 1). 
Figure 1. Long and short lines used in the study

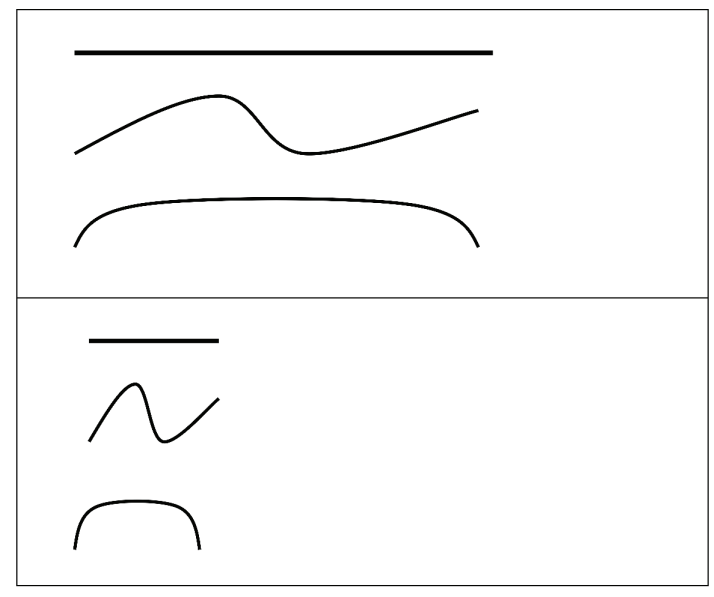

Participants were seated approximately 50 centimeters from the computer screen. Each computer screen had a 17 -inch display with $1024 \times 768$ screen resolution. In the beginning participants had to complete tasks unrelated to the main part of the procedure in order to mask the purpose of the experiment. This part of the experiment included two simple tasks. First, participants were asked to complete five simple Raven's matrices (Raven, 2000). Then, participants had to observe a short sequence of geometrical figures, which appeared one by one. Participants had to set apart those figures from a second sequence of geometrical figures. Depending on the experimental condition participants were asked either to draw or to observe presented lines, which were in short or long format. In the drawing condition, the instruction was to copy the lines as accurately as possible. There was no indication of the scale that participants have to use. They were asked to draw the lines considering their length and shape by using a pen and paper. In the observing condition, participants were asked to observe the lines attentively. After completing the instructions regarding the lines, participants were asked to answer the following question: "What is the length of the Vistula river?". The Vistula river is the longest and the most known river in Poland, resembling in that regard the Mississippi river used in the experiment conducted by Oppenheimer et al. (2008).

\section{Results and discussion}

We used data from 143 subjects, as five participants who figured out the hypotheses of the study were excluded from further analysis. The K-S test showed that the data was not normally distributed $[D=5.716, p<.001]$. The data distribution was violated mostly because of extreme observations (i.e. 0, 50,000 and 300,000 kilometers, in comparison to the actual length of the Vistula river, which is 1047 kilometers). We performed a 5\% mean trim on both sides in order to fit a normal distribution $[D=0.928$, $p=.355]$. Following Oppenheimer et al. (2008) we performed a $2 \times 2$ ANOVA analysis aimed at revealing an interaction of condition and length. The interaction was significant $F(3,123)=2.772, p=.044, \eta^{2}=.063$. In the drawing condition, the average estimate of the Vistula river was 571.48 kilometers after drawing the short lines. Drawing the long lines resulted in a much higher average estimate of 737.66 kilometers. Planned comparisons revealed that the estimations differed significantly between those groups, $t(66)=-1.997, p=.048, d=0.53$. These results are consistent with Hypothesis 1 and replicate findings from previous experiments (Oppenheimer et al., 2008). As predicted, observing the lines was not enough to effectively anchor the estimations. The average estimates were 756.62 kilometers for the short lines and 801.81 kilometers for the long lines. Those estimations did not differ significantly, $t(61)=-0.522$, $p=.602$. Therefore Hypothesis 2 is confirmed (Fig. 2).

Figure 2. Results from Experiment 1. The average estimates differ significantly in the drawing condition. Error bars are +- $S E$

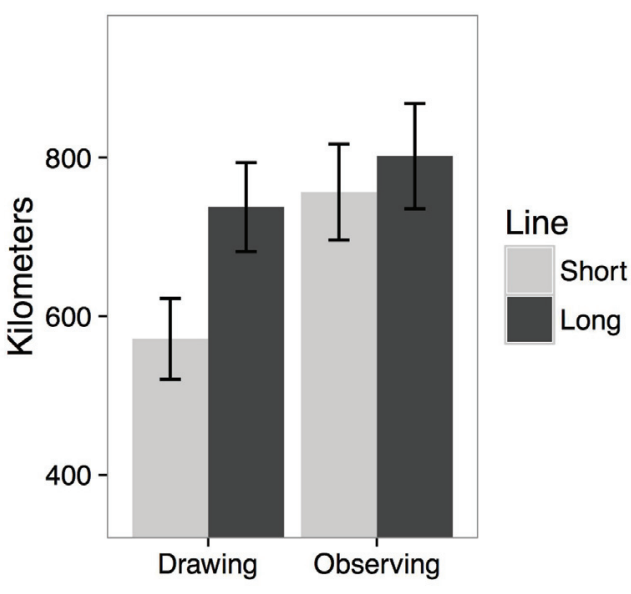

Next, we employed the PROCESS macro (Hayes, 2013) implemented in the SPSS statistical software to perform an indirect effect analysis. We expected that the non-numerical anchor would influence the length of the lines drawn by participants (mediator variable) that in turn would affect numerical estimations. We found that participants who were presented with long lines estimated the Vistula river as longer $(b=264.51, p=.042)$ and reproduced significantly longer lines $(b=79.75, p<.001)$. The length of the reproduced lines, however, did not affect estimations of the river length. Based on bias-corrected bootstrap 95\% confidence intervals (10,000 samples) for the indirect effect, we found that the length of the reproduced lines did not mediate the relationship between the experimental condition and the estimations $(95 \%$ boot CIs[-308.89, 51.83]). Hypothesis 3 was discarded.

Those results suggest that it is not the actual length but the act of drawing itself that matters the most. Drawing the lines demands focus on spatial properties such as length, shape and the relation between the single lines to each other. When the lines are just observed, they are not processed in the same way. We propose that this may be the reason behind creating the sense of "smallness" or "largeness" that could effectively shift the estimations - the explanation proposed by Oppenheimer et al. (2008). 
In Experiment 1, we have shown that the act of drawing was crucial to obtaining the non-numerical anchoring. There was also no relation between the length of the drawn lines represented in millimeters and the final estimations. These results suggest that this kind of anchoring is dependent on the way of processing the stimuli serving as the anchor. The interesting question emerging from those results is whether simply drawing a number is sufficient to anchor the estimations. Predictions based on previous research seem to be unclear. Specifically, if it is the same mechanism that underlies cross-modal anchoring and numerical anchoring, processing a number in order to draw it should be enough to obtain the anchoring effect. Drawing a stimulus enables the magnitude priming, which is the core of the cross-modal anchoring described by Oppenheimer et al. (2008). However, results obtained by Brewer and Chapman (2002) point out that drawing does not suffice to acquire an anchoring effect. If drawing a physical stimulus is enough to process its physical magnitude, than drawing a number should anchor the estimations because of its physical format, but not numerical value. Is it possible to acquire a cross-modal anchoring while using numbers of different physical size and anchor the estimations with no relation to their numerical values?

\section{Experiment 2}

Experiment 1 showed that drawing the lines shifts the estimations, while observing the lines does not have a similar effect. In Experiment 2, we wanted to apply those findings to the anchoring with numerical anchors. Whalen, Gallistel and Gelman (1999) proposed a mechanism of non-verbal counting - which refers to a general ability for transforming unspecified quantities into precise numbers. When a set of stimuli is presented too quickly to count it (i.e. flashes of light) people show a general tendency to make consistent estimations regarding the change of the proportions of given stimuli. Considering previous research we should take into account that numerical stimuli (i.e. set of dots) are considered to be processed nonconsciously and stored in memory as magnitude representations, reflected as precise numbers or general approximations (Dehaene, Bossini, \& Giraux, 1993). Magnitude representations are being retrieved effortlessly, automatically and without awareness (Tzelgov, Meyer, \& Henik, 1992). Moreover, the physical features of stimuli and numerical processing are continuously available to each other. In other words, it is not just the numerical value that is derived from the presented number, but also its physical size. What is more, Sellaro, Treccani, Job and Cubelli (2015) showed in their study that the information about the size of the object is activated regardless to the goal of the task. Based on those results, simply asking to draw the presented number should suffice to acquire an adequate sense of magnitude emerging from the physical size.

Considering the study conducted by Brewer and Chapman (2002) simply presenting or copying a number should not affect the estimations in any way. In Experiment 2 we want to verify, whether using numbers of different physical size would affect the estimations without any relation to their numerical value. Using the same number depicted in two format sizes (small and large) should generate different senses of "smallness" or "largeness", resulting in different estimations within the same number. We designed a new procedure which allows to test our research questions. Similar to the previous experiment, the presented numbers had to be either drawn or observed. The designed procedure used simple cognitive tasks that imposed attention to the processing of the presented anchors.

We expected that in the drawing condition the average estimates will differ between the format conditions: the average estimate in the "large" format condition will be higher then in the "small" format condition (H1). In the drawing condition, we also expect that the estimations will be made regardless of the numerical value: there will be no relation between the numerical values and the estimations $(\mathrm{H} 2)$.

In the observing condition, we expect that the average estimates would not differ between the format conditions: there will be no difference between average estimates in the "large" and the "small" format conditions (H3). Similarly to drawing condition: there will be no relation between numerical values and the estimations (H4).

\section{Method}

Participants

Thirty-four psychology students participated in the experiment in exchange for course credits. Participants provided informed consent before the experiment. The participation in this study was voluntary, anonymous, and in agreement with the guidelines of the Ethical Committee.

\section{Procedure, stimuli and design}

The experiment took place in a room with one computer stand. The computer screen had a 17 inch display with $1024 \times 768$ screen resolution. The procedure was a within-subjects repeated measures design. Each participant went through the drawing and observing conditions both consisting of eight trials. One trial consisted of presenting a number, estimation task and masking task. The observing condition was always presented after the drawing condition. Within a condition, the trials were in random order for each participant (Fig. 3).

We decided that the anchor has to be presented before the estimation target to maintain a similar order to Experiment 1. We chose eight numbers to serve as anchors. Since there is no established way of choosing the numbers to serve as anchors, the numbers were arbitrarily chosen from exponential values of numbers 3 and 6 (the first one, two, three and four-digit numbers: 3, 27, 243, 2187 and 6, 36, 216, 1296). Each number was designed in a "large" (height: 10 centimeters) and "small" (height: 2 centimeters) format. Participants were seated approximately 50 centimeters from the computer screen. In the drawing condition participants were asked to observe the screen attentively in order to perceive a number that was presented for $450 \mathrm{~ms}$. Then they had to draw that number on a piece of paper placed next to 
Figure 3. In this scheme we present an example of one trial. In the beginning of each trial the anchor value in small or large format is presented for $450 \mathrm{~ms}$. The number task depends on the condition: in the drawing condition the number has to be drawn on a piece of paper, in the observing condition participants have to decide whether the number is even or not. Then precisely 30 dots are being presented for $100 \mathrm{~ms}$. Participants are asked to estimate the amount of observed dots. After the estimation task, a string of 14 letters is presented. Participants have to decide if a chosen letter appeared or not.

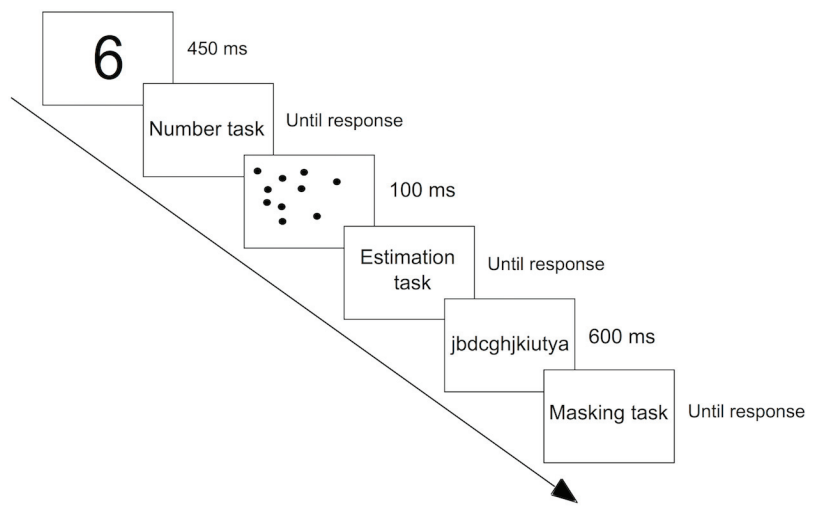

the computer. There was no information regarding the scale that has to be used for drawing the numbers. In this condition presented numbers were 3, 36, 243 and 1296, each in "large" and "small" format. For each number a separate piece of paper was provided. In the observing condition the presented numbers were 6, 27, 216 and 2187. Participants were asked to observe them attentively in order to decide whether the presented number is even or not. In the estimation task participants were asked to estimate the amount of dots presented on the screen. Participants were asked to watch the screen attentively, where a set of thirty dots were presented. Then they were asked about the amount of dots. We designed sixteen different sets of thirty randomly distributed dots. Following Izard and Dehaene
(2008) the dots were present for $100 \mathrm{~ms}$. For the masking task, we designed sixteen different sets of fourteen letters. Participants had to observe a string of letters presented for $600 \mathrm{~ms}$ and answer a question: "Was there a letter ' $\mathrm{x}$ ' in the string?". For every trial the ' $x$ ' became a different letter.

\section{Results and discussion}

As in Experiment 1, the results for drawing and observing conditions will be analyzed separately. We performed a $2.5 \%$ mean trim in order to account for the extreme observations.

\section{Drawing condition}

We used a linear mixed model analysis with subjects as random intercept effects. The analysis revealed a significant main effect of size $F(1,212.791)=8.193, p=.005$. As we predicted, the numbers presented in the "large" format caused higher average estimate $(M=29.38 ; S D=1.44)$ than the "small" format numbers $(M=27.06 ; S D=1.45)$. Also consistent with our assumptions, main effect of the numerical value was not significant $F(3,212.061)=1.768, p=.154$, showing that there was no relation between the numerical values and the average estimates. As presented, the crossmodal anchoring was observed, while the numeric values had no impact on the estimation process. Additionally, we tested for an interaction effect of size and numerical values, which was proved to be significant $F(3,211.921)=3.211$, $p=.024$. We performed planned comparisons in order to verify the possible differences in estimations between "small" and "large" number format for each numerical value separately. In order to avoid the Type I error, we applied the Bonferroni correction in all of the performed comparisons. The analysis showed statistically significant differences for numbers 36, $t(212.216)=-2.603, p=.04, d=0.31$ and $243, t(212.460)=-2.67, p=.032, d=0.37$. The differences for $3 t(211.625)=-1.625, p=.424$ and $1296 t(212.237)=1.149$, $p>.05$ were not significant. (Fig. 4).

Interestingly, in this particular condition the factor of size format mattered only for anchors of 36 and 243 . We hypothesize that numbers such as 3 or 1296 are generally perceived as "small" or "large" without context. Using

Figure 4. Results from the drawing condition of Experiment 2. The average estimates differ significantly in the 36 and 243 condition. Error bars are $+-S E$

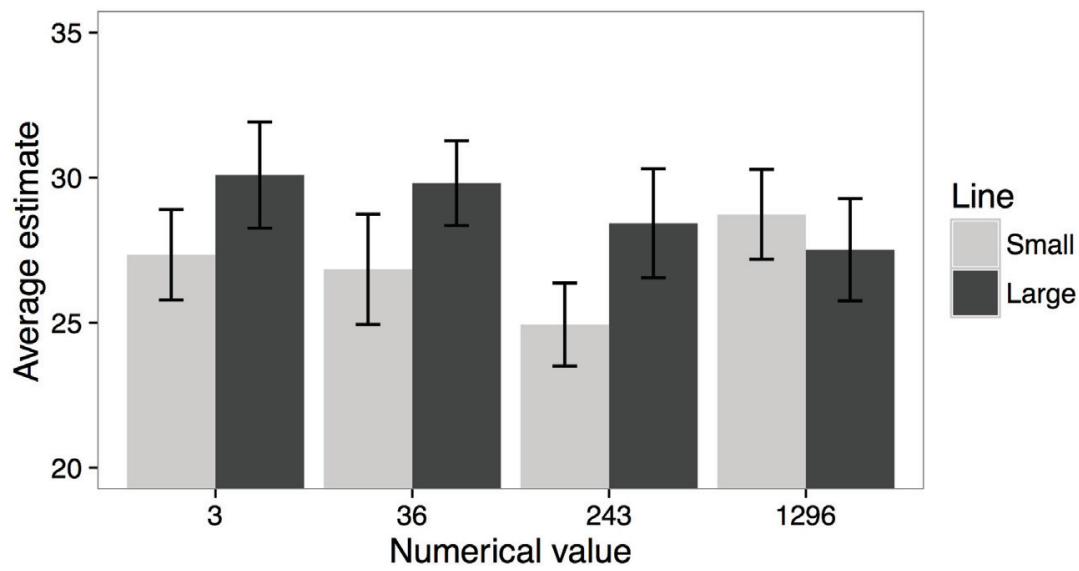


the same format size for every anchor value may have been enough in the case of the 36 and 243 conditions, but presumably number 3 has to be much bigger to generate a sense of "largeness" when number 1296 has to be much smaller to generate a sense of "smallness".

\section{Observing condition}

Analogous to the drawing condition, we applied the linear mixed model analysis with subjects as random intercept effects. Consistent with Hypothesis 3, the main effect of size was not significant $F(1,212.067)=0.173$, $p=.678$. The analysis revealed a significant main effect of the numerical value $F(3,211.806)=5.454, p=.001$. In order to explore the relations between numerical values, we performed the paired comparisons. The results are not completely consistent with Hypothesis 4: differences were found between 27 and other anchor values (Fig. 5).

The average estimate in the 27 anchor condition was significantly lower than the average estimates in the 6 condition $t(212.066)=-3.523, p=0.006, d=0.44$, the 216 condition $t(211.747)=-2.89, p=.024, d=0.38$,

Figure 5. Results from the observing condition of Experiment 2. The average estimate of the 27 condition differs significantly from other conditions. Error bars are +- $S E$

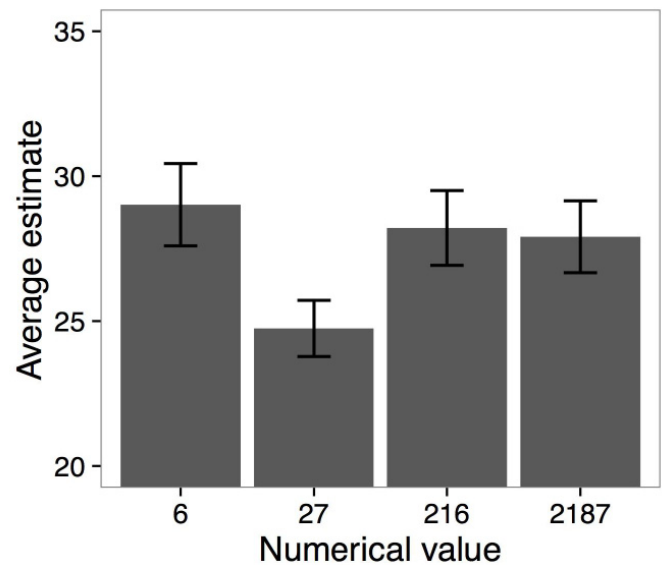

and the 2,187 condition $t(211.769)=-3.36, p=.006$, $d=0.35$.. Yet, since there were no differences between other conditions, we cannot attribute it to the basic anchoring. We suggest that the differences between the 27 and other anchor conditions can be explained by the fact that in each trial, participants were presented with precisely 30 dots. In experiments regarding the mental number line (Izard \& Dehaene, 2008) it is shown that the amount of presented dots is always underestimated. It is probable that 27 was simply a value that served as a possible answer when asked about the amount of dots. The average estimate in the 27 condition has a lower standard deviation $(S D=7.679)$ than the other conditions $(6 S D=11.20,216 S D=10.13$, $2,187 S D=10.23)$. This finding suggests that because of the proximity between numbers 27 (stimuli) and 30 (actual amount of dots) the estimations did not vary as in the other conditions, resulting in a lower average estimate.

Analysis also revealed a significant interaction of numerical value and size $[\mathrm{F}=3.399, \mathrm{p}=.019]$. Yet, after applying the Bonferroni correction, none of the comparisons was found to be significant. The results of the comparisons are $t(212.045)=-2.26, p=.1$ in the 216 condition, $t(211.631)=1.885, p=.244$ in the 6 condition, $t(212.206)=-0.378, p>.999$ in the 27 condition and $t(211.852)=1.278, p=.808$ in the 2187 condition. Those results are clearly not as explicit as those in the drawing condition (Fig. 6).

\section{General discussion}

In our study we investigated the mechanism underlying non-numerical anchoring. Our first finding showed that drawing lines of different lengths effectively shifts numerical estimations, yet the actual length of drawn lines represented in millimeters does not relate to final estimates. Our original research suggests that the act of drawing may be crucial for this phenomenon to occur observing the lines proved to be ineffective. We suggest that the cognitive processes engaged in drawing the stimuli may produce the sense of magnitude and in effect bias the estimations. In Experiment 2 the estimations were not

Figure 6. Results from the observing condition of Experiment 2. No significant differences were found. Error bars are +- $S E$

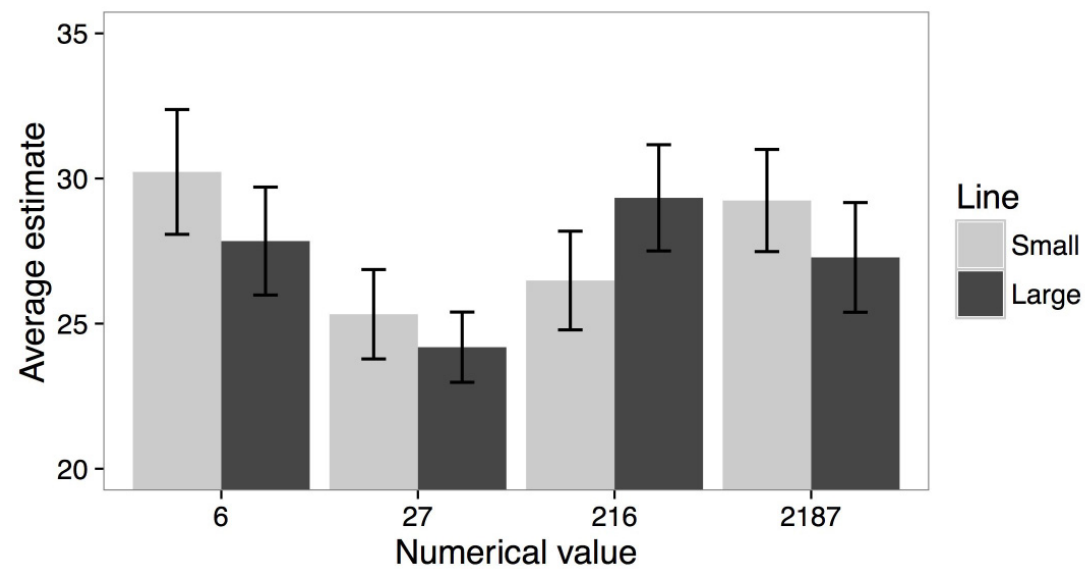


affected by the numerical values of the presented numbers. It was the magnitude priming of physical size that affected the estimations in the drawing condition of Experiment 2. Given that the presented numbers did not influence the estimations, we cannot attribute the obtained results to the basic anchoring effect. Our novel results support the notion that it is not the same psychological mechanism that underlies the basic anchoring and cross-modal anchoring.

In both experiments the act of drawing was a key factor in generating a sense of "smallness" or "largeness". Those results suggest that drawing is determining a different way of processing the presented stimuli. Wilson et al. (1996) claim that the anchoring effect can be obtained if the anchor is given sufficient attention. Other research implies that it is a matter of how deeply the stimuli are processed (Craik \& Lockhart, 1972; Jelicic \& Bonke, 1991). In the case of cross-modal anchoring we suggest that attention focused on spatial properties is necessary to shift the estimations. When copying the lines, it is necessary to consider the length, shape and the relation between the single lines to each other. Moreover, drawing numbers depicted in different format size effectively anchored the subsequent estimations even if the numbers were not drawn consistently with the format size. Following Tzelgov, Meyer and Henik (1992), numerical value and physical features are continuously available to each other. We suggest that this is just the case - the drawing task demands remembering the number, which contains both the numerical value and the format size. We believe that this way of processing generates the sense of magnitude, which in turn effectively affects the estimations. There is evidence that the anchor has to be compatible with the target (Strack \& Mussweiler, 1997; Chapman \& Johnson, 1994) but also that the anchoring effect occurs even when the anchor is not compatible with the target (Strack \& Mussweiler, 1999). While the conclusions regarding the numerical anchoring differ, Oppenheimer et al. (2008) showed that using the magnitude priming allows to achieve a cross-modal anchoring: drawing lines of different length did not only shift the estimations regarding length but also estimations regarding temperature. Our results extend the understanding of non-numerical anchoring, showing that under certain conditions the non-numerical anchoring works while the numerical anchoring does not. This evidence suggests that numerical and non-numerical anchoring are separable, even if based on the same mechanism. Nonetheless, the explanations proposed for the classical anchoring effect focus primarily on the number serving as the anchor. Whether the number is supposed to cause insufficient adjustment (Tversky \& Kahneman, 1974) or has to be presented as information compatible with the estimation task (Strack \& Mussweiler, 1999), the current models do not suffice to explain the crossmodal anchoring. Oppenheimer et al. (2008) suggest that lines serving as anchors generate a sense of "smallness" or "largeness". We extend this explanation by proposing a mechanism that takes into account number and spatial processing, resulting in transforming magnitudes to numbers. Specifically, we consider a relation between research regarding mental number line and quantity perception. Mental number line describes a spatial orientation of number magnitude (Dehaene et al., 1993). Numbers are placed on a metaphorical line, oriented from left to right in a growing order. Research has shown that there is a connection between numbers and visuo-spatial representations (Zorzi, Priftis, \& Umiltà, 2002). When performing tasks involving the mental number line and physical line bisection the observed bias is consistent, which suggests that hemispheric asymmetries in spatial attention operate similarly in physical and numerical space (Longo \& Lourenco, 2006). The similarity in processing of the mental number line and physical lines implies a relation between spatial properties and numbers, especially that greater bias on the line bisection task related to greater bias on the mental number line. In the same vein, we suggest that magnitudes derived from physical stimuli may be differentiated just like in the case of the mental number line. Given evidence that the generalized magnitude representations are a fundamental mental ability which may work across the sensory modalities (for review see Lourenco \& Longo, 2011) we suggest that the representations of magnitude and physical features are interrelated.

Research supports a notion of abstract coding of numerical magnitude. Activation of the horizontal segment of the intraparietal sulcus (hIPS) is connected to performing number-processing tasks (Dehaene, Piazza, Pinel, \& Cohen, 2003). Coding of quantities in hIPS is not dependent on the notation - numbers represented as dots, digits or number words have the same neural representation (Piazza, Pinel, Le Bihan, \& Dehaene, 2007). Those findings support a mechanism of non-verbal counting (Whalen et al., 1999) and explain how is it possible to generate numbers without counting. Yet, as claimed by Whalen et al. (1999), values derived from the same magnitudes may differ because of memory noise, which distorts the process. That is consistent with the fact that the mental number line is most often not calibrated (Izard \& Dehaene, 2008). The estimations of the quantity of presented dots were either inconsistent or biased by the number provided with the first set of dots. We argue that in case of cross-modal anchoring a magnitude derived from the anchor affects the magnitude generated to answer the subsequent question. Sleeth-Keppler (2013) showed that it is possible to obtain the anchoring effect with words serving as anchors. Vague descriptions of quantity such as „many" or „few” had a substantial impact on the estimations. According to Sleeth-Keppler (2013) the activation of the given word influences the subjective expected value of an estimate, shifting the range of plausible values towards the lower or upper bound. We suggest that magnitude priming obtained with physical stimuli is based on similar mechanism - because of magnitude priming the expected value in the estimation task is biased towards "largeness" or "smallness". We suggest that within the sense of magnitude there is a range of possible values that might be derived from it. The estimation task provides a certain context, which allows to 
place the unspecified sense of magnitude on a certain scale and transform it into a precise number. Our proposition is consistent with previously proposed models - the process of insufficient adjustment (Tversky \& Kahneman, 1974) and the Scale Distortion Theory (Frederick \& Mochon, 2011). According to the Scale Distortion Theory in context of a small anchor, large numbers which are placed on the same scale feel larger. In case of non-numerical anchoring the initial magnitude representation derived from the physical size of the stimuli serves as a starting point for subsequent adjustment process. The estimations are placed on a certain scale, yet the perception of the values placed on the scale differs depending on the primed magnitude. In result, because of the sense of „smallness" the higher values on the scale seem to be inadequately big in terms of magnitude, while the opposite effect occurs in case of the sense of ,largeness".

Considering future research it is worth to mention the embodiment approach to cognition (for example Wilson, 2002). According to the embodiment theories there is a deep connection between the cognitive processes and the interaction between the body and the environment. This approach would be particularly useful in exploring the possible mechanisms and contexts underlying the possibility of generating the sense of magnitude. Perhaps the task of drawing the lines is simply not sufficient to capture the complexity behind the generated sense of magnitude and engaged cognitive processes. It is possible that using a different procedure than Oppenheimer, LeBoeuf and Brewer (2008) would allow to discover a significant mediation effect between the generated sense of magnitude and the magnitude of the estimations.

Given the results presented in the article it is crucial to verify if the same rules apply to numerical and nonnumerical anchoring when the comparative question is asked. Referring to considerations regarding the judgmental strategies made by Strack and Mussweiler (1997) it is also necessary to establish whether the same process is used to generate an absolute answer when the estimation targets differ. In most studies regarding anchoring effects, the target of the estimations is well known (i.e. the existence of Vistula river), but its properties are not usually a part of general knowledge (the exact length of the Vistula river). Yet, it is plausible that in order to estimate the length of a river, several cues are retrieved from memory to facilitate the whole process. Notions such as "the longest river in Poland", "goes through the whole country from South to North" or an additional comparative question: "If I know that there is a river in Poland that is about $800 \mathrm{~km}$ long, how long is the Vistula river?" might effectively narrow the set of values considered as plausible answers and result in distortion of the anchoring effect. Such hypotheses, however, need further verification.

Contrary to previous findings, Ciritcher and Gilovich (2008) proposed that basic anchoring is not as fragile as suggested in prior research, stating that potential anchor values present in the environment may affect the numerical judgment. In their study, participants were asked to estimate how much they would spend at a restaurant, showing that the answers were influenced by whether the restaurant was named "Studio 19" or "Studio 97". The effect was observed because the anchor was present during the estimation task, while Wilson et. al. (1996) used anchoring task and estimation task that were not related. This form of basic anchoring based on incidental anchors shows that it is possible to effectively shift the estimations without the comparative question. The relation between incidental anchoring and the non-numerical anchoring is yet to be examined, seeming to be another promising direction of future research.

In sum, the presented study shows that the estimation shift can be obtained with magnitude priming which can be derived from the physical features of the number, regardless of the actual numerical value. Our results suggest that the cross-modal anchoring is separable from the anchoring based on numerical values and possibly requires a different theoretical approach or extending existing ones.

\section{References}

Brewer, N.T., \& Chapman, G.B. (2002). The fragile basic anchoring effect. Journal of Behavioral Decision Making, 15, 65-77.

Chapman, G.B., \& Johnson, E.J. (1994). The limits of anchoring. Journal of Behavioral Decision Making, 7, 223-242.

Craik, F.I., \& Lockhart, R.S. (1972). Levels of processing: A framework for memory research. Journal of Verbal Learning and Verbal Behavior, 11(6), 671-684.

Critcher, C.R., \& Gilovich, T. (2008). Incidental environmental anchors. Journal of Behavioral Decision Making, 21(3), 241-251.

Dehaene, S., Bossini, S., \& Giraux, P. (1993). The mental representation of parity and number magnitude. Journal of Experimental Psychology: General, 122(3), 371 .

Dehaene, S., Piazza, M., Pinel, P., \& Cohen, L. (2003). Three parietal circuits for number processing. Cognitive Neuropsychology, 20(3-6), 487-506.

Frederick, S.W., \& Mochon, D. (2011). A scale distortion theory of anchoring. Journal of Experimental Psychology: General, 141(1), 124.

Friedlander, M.L., \& Stockman, S.J. (1983). Anchoring and publicity effects in clinical judgment. Journal of Clinical Psychology, 39, 637-643.

Furnham, A., \& Boo, H. C. (2011). A literature review of the anchoring effect. The Journal of Socio-Economics, 40(1), 35-42.

Gilovich, T., Griffin, D., \& Kahneman, D. (Eds.). (2002). Heuristics and biases: The psychology of intuitive judgment. Cambridge: Cambridge University Press.

Hayes, A.F. (2013). Introduction to mediation, moderation, and conditional process analysis: A regression-based approach. New York, NY: Guilford Press.

Higgins, E.T., Rholes, W.S., \& Jones, C.R. (1977). Category accessibility and impression formation. Journal of Experimental Social Psychology, 13(2), 141-154.

Higgins, E.T., \& Brendl, C.M. (1995). Accessibility and applicability: Some" activation rules" influencing judgment. Journal of Experimental Social Psychology, 31(3), 218-243.

Izard, V., \& Dehaene, S. (2008). Calibrating the mental number line. Cognition, 106(3), 1221-1247.

Jelicic, M., \& Bonke, B. (1991). Level of processing affects performance on explicit and implicit memory tasks. Perceptual and Motor Skills, $72(3 \mathrm{c}), 1263-1266$.

Kahneman, D., \& Knetsch, J. (1993). Anchoring or shallow inferences: The effect of format. Unpublished manuscript.

Kaustia, M., Alho, E., \& Puttonen, V. (2008). How much does expertise reduce behavioral biases? The case of anchoring effects in stock return estimates. Financial Management, 37(3), 391-412.

Klayman, J., \& Ha, Y.W. (1987). Confirmation, disconfirmation, and information in hypothesis testing. Psychological review, 94(2), 211. 
Kristensen, H., \& Garling T. (1997). The effects of anchor points and reference points on negotiation process and outcome. Organizational Behavior and Human Decision Processes, 71, 85-94.

LeBoeuf, R.A., \& Shafir, E. (2006). The long and short of it: Physical anchoring effects. Journal of Behavioral Decision Making, 19(4), 393-406.

Longo, M.R., \& Lourenco, S.F. (2006). On the nature of near space: Effects of tool use and the transition to far space. Neuropsychologia, 44(6), 977-981.

Lourenco, S.F., \& Longo, M R. (2011). Origins and development of generalized magnitude representation. Space, time, and number in the brain: Searching for the foundations of mathematical thought, 225-244.

Mussweiler, T., \& Strack, F. (1999). Hypothesis-consistent testing and semantic priming in the anchoring paradigm: A selective accessibility model. Journal of Experimental Social Psychology, 35, 136-164.

Northcraft G.B., \& Neale, M.A. (1987). Experts, amateurs, and real estate: An anchoring-and-adjustment perspective on property pricing decisions. Organizational Behavior and Human Decision Processes, 39, 84-97.

Oppenheimer, D.M., LeBoeuf, R.A., \& Brewer, N.T. (2008). Anchors aweigh: A demonstration of cross-modality anchoring and magnitude priming. Cognition, 106(1), 13-26.

Piazza, M., Pinel, P., Le Bihan, D., \& Dehaene, S. (2007). A magnitude code common to numerosities and number symbols in human intraparietal cortex. Neuron, 53(2), 293-305.

Quattrone, G.A., Lawrence, C.P., Warren, D.L., Souza-Silva, K., Finkel, S.E., \& Andrus, D.E. (1984). Explorations in anchoring: The effects of prior range, anchor extremity, and suggestive hints. Unpublished manuscript, Stanford University, Stanford, CA.

Raven, J. (2000). The Raven's progressive matrices: change and stability over culture and time. Cognitive Psychology, 41(1), 1-48.
Seron, X., Pesenti, M., Noël, M.P., Deloche, G., \& Cornet, J.A. (1992). Images of numbers, or "When 98 is upper left and 6 sky blue". Cognition, 44(1), 159-196.

Strack, F., \& Mussweiler, T. (1997). Explaining the enigmatic anchoring effect: Mechanisms of selective accessibility. Journal of Personality and Social Psychology, 73, 437-446.

Mussweiler, T., \& Strack, F. (1999). Hypothesis-consistent testing and semantic priming in the anchoring paradigm: A selective accessibility model. Journal of Experimental Social Psychology, 35(2), 136-164.

Sellaro, R., Treccani, B., Job, R., \& Cubelli, R. (2015). Spatial coding of object typical size: evidence for a SNARC-like effect. Psychological research, 79(6), 950-962.

Sleeth-Keppler, D. (2013). Taking the high (or low) road: a quantifier priming perspective on basic anchoring effects. The Journal of social psychology, 153(4), 424-447.

Tversky, A., \& Kahneman, D. (1974). Judgment under uncertainty: Heuristics and biases. Science, 185(4157), 1124-1131.

Tzelgov, J., Meyer, J., \& Henik, A. (1992). Automatic and intentional processing of numerical information. Journal of Experimental Psychology: Learning, Memory, and Cognition, 18(1), 166.

Whalen, J., Gallistel, C.R., \& Gelman, R. (1999). Non-verbal counting in humans: The psychophysics of number representation. Psychological Science, 10(2), 130-137.

Wilson, M. (2002). Six views of embodied cognition. Psychonomic bulletin \& review, 9(4), 625-636.

Wilson, T. D., Houston, C.E., Brekke, N., \& Etling, K.M. (1996). A new look at anchoring effects: Basic anchoring and its antecedents. Journal of Experimental Psychology: General, 125, 387-402.

Wong, K., \& Kwong, J. (2000). Is $7300 \mathrm{~m}$ equal to $7.3 \mathrm{~km}$ ? Same semantics but different anchoring effects. Organizational Behavior and Human Decision Processes, 82, 314-333.

Zorzi, M., Priftis, K., \& Umiltà, C. (2002). Brain damage: neglect disrupts the mental number line. Nature, 417(6885), 138-139. 